\title{
Optimal control of transistor SRM converters with reduced number of switching element
}

\author{
László Számel \\ Budapest University of Technology and Economics/Department of Power Engineering, Budapest,
}

\begin{abstract}
Nowadays switched reluctance motor (SRM) drives have been widely used in the field of controlled electric motor drives. The paper proposes a simple control method for SRM drives. Current control of the reduced switching item number SRM drives contain modified current reference signal. This method provides that torque ripple would not be significantly higher at a Miller-kind $(n+1)$ transistor converter controlled n-phase drive - in other words a reduced switching item number SRM converter feed - than at independent current control in each phases carried out with $2 n$ transistors.
\end{abstract}

\section{INTRODUCTION}

Nowadays switched reluctance motor (SRM) drives have been widely used in the field of controlled electric motor drives. Switched reluctance motors are doublesalient, single excited motors. This means that the motor has salient poles an both the rotor and the stator, but only the stator carries windings. The motor does not require special material, e.g. magnet, it is simply built up from a stack of salient pole laminations.

\section{DRIVE SYSTEM}

The block scheme of the examined drive system is shown in Fig. 1.

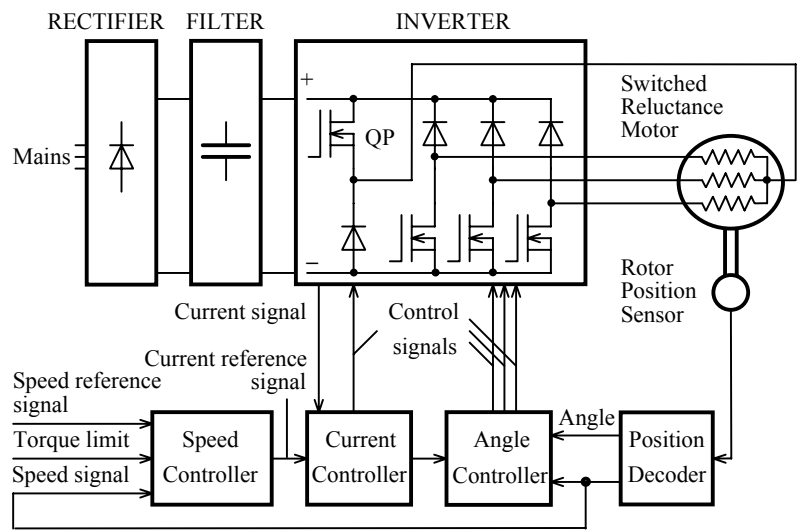

Fig. 1. Block scheme of drive system.

The supply unit consists of three main blocks, namely the RECTIFIER, the FILTER and the INVERTER. The inverter is a pulsed width modulated (PWM) one, marked with QP in the figure and it contains one-one switching transistor per phase and a brake chopper, not shown in the figure. The common point of phase windings is supplied by the PWM inverter. It is of autonomous operation and has an inner current control loop. The other ends of phase windings are connected to the phase switching transistors.

It follows from the operational principle of SRM that its phase windings are to be excited at a well determined angle of the rotor position in an appropriate order. This is why a Rotor Position Sensor is to be mounted on the shaft of the motor. In our case the position sensor is a resolver. It can be calculated from the pole numbers that the phase switchings have to follow each other by 15 degree. The resolver is supplied by an oscillator circuit, their signals are evaluated by a Position Decoder.

The Position Decoder has two outputs: the Angle and Speed signals. Based on the two signals, the Angle Controller composes the Control signals for the phase switching transistors.

Fundamentally, SRM drives have two control loops, the outer one is the speed loop, Speed Controller and the inner one is the current loop, Current Controller. The output signal of the Speed Controller serves for a Current reference signal of the Current Controller. The hardware and software tools together fulfill the two-loop control. The Current Controller produces the control signal for the PWM inverter, and receives the Current signal from the PWM inverter at the same time.

\section{OPERATION OF THE SRM}

The simplified operation principle of the SRM is as follows: When current is passed through the phase (stator) windings the rotor tends to align with the stator poles, that is it produces a torque that tends to move the rotor to a minimum-reluctance position. When a rotor pole is approaching the aligned position of the excited stator pole, positive (motoring) torque is produced, regardless of the direction of the current.

The terminal voltage $u$ on the phase winding can be expressed with the following equation:

$$
u=i R+L \frac{d i}{d t}+i \omega \frac{d L}{d \Theta},
$$

where:

$i$ is the phase current,

$R$ is the resistance of phase,

$L$ is the incremental inductivity of phase winding,

$\Theta$ is the rotor angle,

$\omega$ is the angular speed of the motor.

The last member of (1) is the inner voltage of the motor which depends on the speed, the phase current and the slope of inductivity curve [2]. 
From [2] the torque $\tau$ of SRM:

$$
\tau=\frac{1}{2} i^{2} \frac{d L}{d \Theta} .
$$

Though (2) is only valid for the linear case, two features follow from it. One of them is that the torque is independent of the sign of current. The other one is that the sign of torque is determined by the slope of inductivity curve.

In Fig. 2 the upper curve signed $\mathrm{L}$ is the so-called inductivity profile which shows the inductivity of phase A in an idealized form as a function of the rotor shaft angle. (The airgap varies with the rotation of laminated rotor with salient poles.) The inductivity profile is determined fundamentally by the motor construction.

The operation of SRM is illustrated in Fig. 2. Phases currents A, B, C are represented by the continuous curves marked by $\mathrm{A}, \mathrm{B}, \mathrm{C}$ respectively in the motoring operation mode. In the generating operation mode the dotted section of curve A is valid.

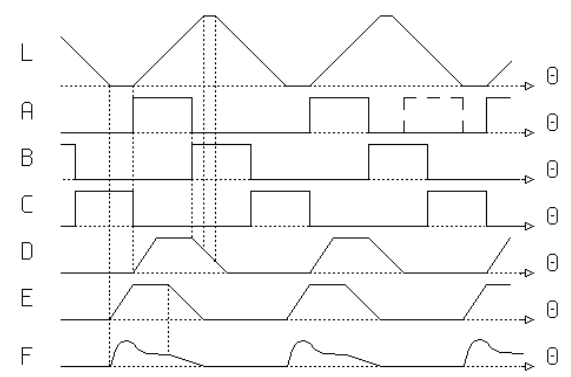

Fig. 2. The inductivity profile and the three-phase currents as a function of the rotor angle position

At high speed phase current A e.g. varies according to curve D in Fig. 2, due to the fact that the period of the current pulse can be compared with the magnetizing, resp. demagnetizing period of the inductance (after switching off the IGBT transistor the current is conducted by the diode). This results in a torque pulsation, because the current is not constant, on the one hand, and the current pulse spans to the negative section of the inductance profile of curve L in Fig. 2, on the other hand. On this section of curve $\mathrm{L}$ the positive torque turns negative. The effect of this detrimental phenomenon can be decreased by advancing the turn-on angle, as shown by curve $\mathrm{E}$ in Fig. 2. At higher speed the current varies according to curve $F$ in Fig. 2 because of the increased role of inner voltage (back EMF).

\section{THE $(\mathrm{N}+1)$ SWITCH CONVERTER}

The $(n+1)$ switch converter shown in Fig. 1 has been reported by T. Miller et al. [3,4]. Operating modes of $(n+1)$ switch converter are analogous to those of the classic converter [5]. When one of the phase switches and common switch QP are turned on during magnetization period, the dc link voltage is applied to the phase winding. During forced demagnetization period, the phase switch and common switch QP have to be turned off; one of the phase diodes and common diode conduct and the magnetic energy is transferred to the capacitor $\mathrm{C}$.

Main drawback of the converter with $(n+1)$ switching element is that phases can not be controlled independently from each other. When the next conducting phase turns on during demagnetization of a phase common QA switching element must be switched on. However in this case current of phase under demagnetization is short-circuited through the common switching element and the diode belonging to that phase. So demagnetization slows down significantly. This problem is especially important when the current of the phase turned off is even less reduced until the slope of the inductance profile becomes negative. At this time the back EMF of the motor further increases the current of the turned-off phase through the common QA switching element in turned on state. At high speed applications this problem requires introduction of additional apparatuses. With the addition suggested by Professor M. Ehsani [5] phase diodes are not directly connected to the common DC link but through a resistance connected in parallel with a switching element. Generally this switching element is turned on. In such a case operation is fully identical to the principle of converter with $(n+1)$ switching element. As far as the turned-off phase is on the negative-slope inductance domain the supplementary switching element turns off and the phase current stops according to electric time constant reduced by the inserted resistance. So the circuit operates with additional losses for a short time and the number of the switching elements has increased to $(n+2)$.

Let us examine closely the approximate demagnetizing process of the converter with $(n+1)$ switching elements at the decreasing-inductance (generator) domain!

The average voltage connected to the turned-on phase neglecting the winding resistance - keeps balance with the back EMF of the SRM so approximating the profile of the inductance linearly changing $(\Delta \mathrm{L} / \Delta \Theta=$ constant $)$ :

$$
u=b_{i} U_{d}=i_{r}(1-k) \omega \frac{\Delta \mathrm{L}}{\Delta \alpha}
$$

where $U_{d}$ is the DC link voltage, $b_{i}$ is the duty cycle of the common switching element, $i_{r}$ is the current reference, $k$ is the ratio between the turned-off phase current and current reference with value between 0 and 1. Equation (3) takes into consideration that current in the turned-on phase is (1$k) i_{r}$ at $i_{r}$ reference value.

For phase turned off and under demagnetization the next equations can be set up at the decreasing-inductance domain:

$$
0=-k i_{r} \omega \frac{\Delta \mathrm{L}}{\Delta \Theta}+L \frac{\mathrm{d} i}{\mathrm{~d} t}+R i
$$

when the common switching element is turned on, and

$$
-U_{d}=-k i_{r} \omega \frac{\Delta \mathrm{L}}{\Delta \Theta}+L \frac{\mathrm{d} i}{\mathrm{~d} t}+R i
$$

when the common switching element is turned off. Equations (4) and (5) contains $(\Delta \mathrm{L} / \Delta \Theta)$ absolute value of the slope of the inductance profile.

The average voltage on the turned-off phase according to $b_{i}$ switching-on rate determined by the switched-on phase is the next:

$$
-U_{d}\left(1-b_{i}\right)=-k i_{r} \omega \frac{\Delta \mathrm{L}}{\Delta \Theta}+L \frac{\mathrm{d} i}{\mathrm{~d} t}+R i
$$


Substituting $b_{i}$ from (3) into (6) we get:

$$
-U_{d}+i_{r} \omega \frac{\Delta \mathrm{L}}{\Delta \Theta}=L \frac{\mathrm{d} i}{\mathrm{~d} t}+R i
$$

According to (7) it can be seen that the condition of current reduction in the turned-off phase at generator domain is:

$$
U_{d}>i_{r} \omega \frac{\Delta \mathrm{L}}{\Delta \Theta}
$$

in other words there is a revolution number (angular speed) limit until the inequality is true.

It is worth to compare the voltages in turned-off phase determining the motor current (in other words the signed sum of the terminal voltage and the motor back EMF) at the two circuits on the positive and negative slope inductance domain. Voltage valid for positive-slope inductance domain of the $(n+1)$ converter can be got similarly to proof (3)-(8) with the difference that the motor back EMF has positive sign in equations (4) and (5). While at $2 \mathrm{n}$ converter the voltage equation can be directly set up because of the independence of the phases. According to these terms the studied voltages are the next:

TABLE I.

VOLTAGES DETERMINING THE CURRENT OF TURNED-OFF PHASE

\begin{tabular}{|c|c|c|}
\hline$\frac{\mathrm{d} \mathrm{L}}{\mathrm{d} \Theta}>0$ & $-U_{d}-k i_{r} \omega \frac{\Delta \mathrm{L}}{\Delta \Theta}$ & $-U_{d}+(1-2 k) i_{r} \omega \frac{\Delta \mathrm{L}}{\Delta \Theta}$ \\
\hline$\frac{\mathrm{d} \mathrm{L}}{\mathrm{d} \Theta}<0$ & $-U_{d}+k i_{d} \omega \frac{\Delta \mathrm{L}}{\Delta \Theta}$ & $-U_{d}+i_{r} \omega \frac{\Delta \mathrm{L}}{\Delta \Theta}$ \\
\hline
\end{tabular}

According to Table 1 next statements can be taken:

- The highest negative voltage reduces current in the turned-off phase is at positive-slope inductance domain of $2 \mathrm{n}$ converter while the lowest available value is at negative-slope inductance domain of $(n+1)$ converter.

- After switching-over from the positive-slope domain to the negative-slope domain by $2 k i_{r} \omega \frac{\Delta \mathrm{L}}{\Delta \Theta}$ less negative voltage is ready for operation at a given converter. This is because the back EMF of the motor changes its sign jump-like in the turned-off phase.

- Compared to $2 \mathrm{n}$ converters by $(1-k) i_{r} \omega \frac{\Delta \mathrm{L}}{\Delta \Theta}$ less negative voltage is ready for operation at $(n+1)$ converters. This voltage comes from the interaction between the turned-on phase and the other phase conducting $(1-k) i_{r}$ current.

So when using $(n+1)$ converter demagnetization of the turned-off phase is slower than at $2 \mathrm{n}$ converters. Slowing down of the demagnetization is not permanent; there is no difference in the voltage at the beginning of the turningoff and higher difference comes at the end of the turningoff process. However it is also provable that negative voltage needed to demagnetization can be provided in very wide speed range. Although at higher speeds limitation of load or application of complementary circuit (for example $(n+2)$ converter) may be needed in order to speed up demagnetization [5].

\section{RIPPLE REDUCED CONTROL METHOD}

The ripple free operation can be realize only with an current waveform depending on the angle, speed and torque [6]. The proposed ripple reduced method changes only the turn-on and the turn-off angle in function of the speed and current reference. The optimum turn-on and turn-off angles of the SRM drive has been determined by computer simulation based on the measured results of the analysed drive. The optimum solution has been fulfilled by four cycles embedded into each other. Two outer cycles give the current and speed reference signals, while two inner ones provide the turn-on and turn-off angles. By this one-one optimum angle pair can be determined to all operating points.

It can be considered an interesting result that the criteria of the minimum torque pulsation does not provide an optimum solution in all cases. The torque pulsation will be minimum in the speed-current plane only in that case if the torque of the motor is relatively small. For this reason a good result can be achieved in such a way if the relative, i.e. compared to the torque of motor, torque pulsation is minimized.

The angle control of the drive determines the actual turn-on and turn-off angles with a two-variable interpolation from the results stored in a look-up table and calculated by the above method.

\section{MODIFICATION OF THE CURRENT CONTROL}

The detriment of the converter with $(n+1)$ switching element is that the torque pulsation can be decreased in a smaller degree by changing the turn-on and turn-off angles.

There are two reasons for this. First of all the effect as it can be seen on Table 1 is that the current in phase turned off is ended by less voltage during longer time. This effect has less importance. Partly because at lower speeds even in this case significant voltage reserve is available and on the other hand this slowing down rather appears only at lower currents in the phases turned-off where the torque production is significantly lower according to it. The other effect is that not only turning-on process effects on turning-off but vice versa turning-off effects on turning-on as well. This problem has not been investigated in the literature yet. The possible cause of this can be that the first effect comes from the topology of the converter while the second one closely hangs together with the control.

Namely, in the case of the constant current reference signal the current increase is limited by the switched-off, but conducting phase current as the regulator controls the sum of two phase currents. The increase of the phase current at starting the conducting state can be forced by the modification of the current reference signal:

$$
i_{r}=y \sum_{j=1}^{3} C_{j}+\sum_{j=1}^{3}\left(1-C_{j}\right) \cdot i_{j}
$$

where:

$i_{r} \quad$ is the current reference signal, 
$i_{j} \quad$ is the current signal of phase $j$,

$y \quad$ is the output of the speed controller,

$C_{j} \quad$ is the control signal of phase $j$ ( 0 or 1$)$.

The supplement of the first member of (9) makes the overlap of the phase conduction possible, while the effect of second member is to increase the reference signal with the current of the switched-off, but not current-free phase.

However modified, increased current reference signal that supports turning-on process effects back the current in phase under turning-off. Under the effect of the increased current reference signal the value of the negative voltage that ends current in the phase turned off further falls. The value of this fall is identical to the inner voltage increase with value of $k i_{r} \omega \frac{\Delta \mathrm{L}}{\Delta \Theta}$ coming from the increased current. So the voltages are the next:

TABLE II.

VOLTAGES DETERMINING THE CURRENT OF TURNED-OFF PHASE

\begin{tabular}{|c|c|c|}
\hline & $(\mathrm{n}+1)$ converter & $\begin{array}{c}(\mathrm{n}+1) \text { converter } \\
\text { with modified current } \\
\text { reference }\end{array}$ \\
\hline$\frac{\mathrm{d} \mathrm{L}}{\mathrm{d} \Theta}>0$ & $-U_{d}+(1-2 k) i_{r} \omega \frac{\Delta \mathrm{L}}{\Delta \Theta}$ & $-U_{d}+(1-k) i_{r} \omega \frac{\Delta \mathrm{L}}{\Delta \Theta}$ \\
\hline$\frac{\mathrm{d} \mathrm{L}}{\mathrm{d} \Theta}<0$ & $-U_{d}+i_{r} \omega \frac{\Delta \mathrm{L}}{\Delta \Theta}$ & $-U_{d}+(1+k) i_{r} \omega \frac{\Delta \mathrm{L}}{\Delta \Theta}$ \\
\hline
\end{tabular}

According to Table 2 it can be seen that with increased current reference signal the most disadvantageous situation is also can be found on the negative-slope inductance section, the value of the voltage: $-U_{d}+(1+k) i_{r} \omega \frac{\Delta \mathrm{L}}{\Delta \Theta}$. At maximum speeds that the drive can reach voltage determining the current in phase turned off can reach even the value of the DC link voltage (at $\mathrm{k}=1$ ). This can cause fast current change with opposite direction than wanted. It must be inhibited.

Smaller positive DC voltage also can not be allowed, as in this case current in the phase turned off increases. According to equation (9) this increases the current reference signal. Under effect of the higher current reference signal duty cycle of the common transistor $\left(b_{i}\right)$ also increases and so there is even higher voltage at the phase turned off. So positive feedback emerges which makes the current uncontrollable. Therefore modified current reference signal is only applicable at lower speeds. In order to secure operation application of modified current reference signal above $30-40 \%$ of the nominal speed is not expedient.

Summarizing we can find out that with current reference signal compensation described by equation (9) torque ripple reduction is possible exactly in that low speed range where it is mostly needed. Nevertheless the suggested compensation also has two disadvantageous properties. First of all, current increases in the common transistor of the converter. Secondly measuring currents in each phase are needed. Accordingly application of this compensation is practical after exhaustive consideration of technologic requirements and costs.

\section{SPEED CONTROL}

Based on the Speed reference signal, the Speed Controller produces the Current reference signal taking the Speed signal and the Torque signal into account. The digital output signal determined by the software is converted by a D/A converter to an analog reference signal The software defines the connection between the current and torque.

The speed feedback signal is determined by calculating the difference between the actual position value and the previous one of the resolver to digital converter. The measuring period ensures an accuracy of $\pm 5 \mathrm{rpm}$. The controller is of PDF-type (an integral element with Proportional and Derivative Feedback).

The control algorithm is as follows:

$$
\begin{aligned}
& y(n \mathrm{~T})=y(n \mathrm{~T}-\mathrm{T})+\mathrm{K}_{\mathrm{i}} e(n \mathrm{~T})+\mathrm{K}_{\mathrm{p}}[\omega(n \mathrm{~T}-\mathrm{T})-\omega(n \mathrm{~T})]+ \\
& \mathrm{K}_{\mathrm{d}}[2 \omega(n \mathrm{~T}-\mathrm{T})-\omega(n \mathrm{~T})-\omega(n \mathrm{~T}-2 \mathrm{~T})]
\end{aligned}
$$

where:

$\mathrm{T}$

$y(n \mathrm{~T})$

$e(n \mathrm{~T})$

$\omega(n \mathrm{~T})$

$(n \mathrm{~T}-\mathrm{T})$

$\mathrm{K}_{\mathrm{p}}, \mathrm{K}_{\mathrm{i}}, \mathrm{K}_{\mathrm{d}}$

The above PDF algorithm can be applied to control the speed of electrical drives, as it makes an optimal adjustment possible for a step change in both the reference signal and the load [7].

The output signal of speed controller can be used preferably for torque command signal to ensure fast dynamics. One solution for the above task is to insert a torque control loop between the speed control loop and the current one. The formation of an actual torque signal makes the torque control complicated and expensive. Knowing the intermediate dc voltage and dc current, the speed and the efficiency, the approximate calculation of the torque is possible [1]. Because of the above difficulties, other, simpler and more effective methods have been analysed. Their common characteristics are that fast dynamics are ensured by applying a constant gain in the speed control loop.

Neglecting the saturation, the motor torque is proportional to the square of current. This means that the current reference signal can be composed from the torque reference signal, produced by the speed controller by the help of a square-root function after composing its absolute value. The motoring and generating operation modes of the drive can be determined from the sign of torque reference signal and the momentary direction of rotation. The change of operation mode is fulfilled by the Angle Controller, illustrated by the arrow between the Current Controller and Angle Controller in Fig.1. 

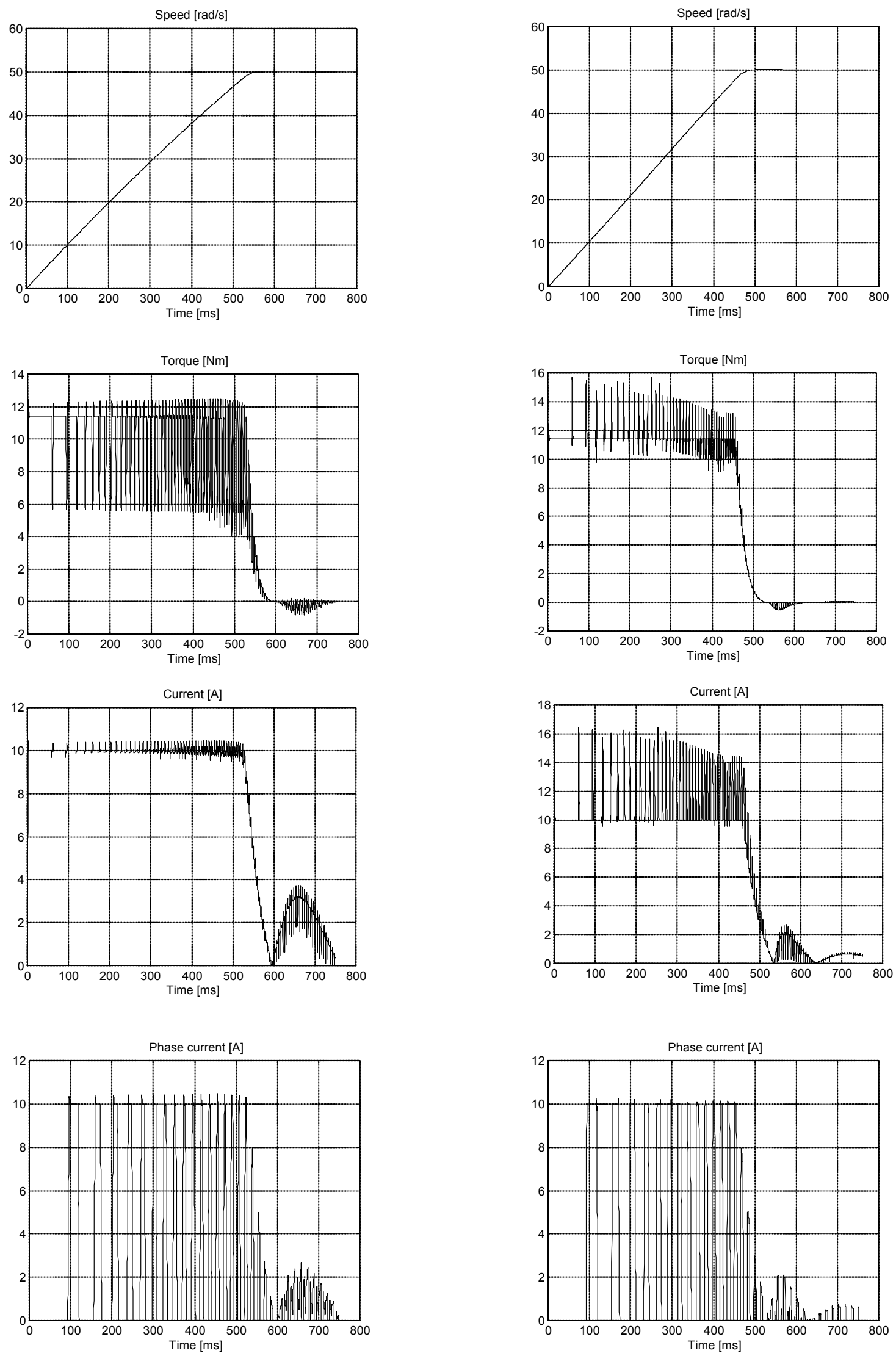

Fig. 3. Simulation results with constant turn-on and turn-off angles

Fig. 4. Simulation results with turn-on and turn-off angles depending on speed and current reference 


\section{SiMULATIONS RESULTS}

In Fig. 3 and Fig. 4 two of many executed simulations are shown. Fig. 3 shows the run-up with constant turn-on and turn-off angles without current reference compensation, while Fig. 4 with turn-on and turn-off angles depending on the speed and current reference and with current reference compensation (9).

\section{TEST RESUlts}

The tests were completed by the described drive system. The test results have supported our theoretical investigations. The oscillograms in the following figures illustrate some typical starting curves and wave forms. The loading machine was a DC motor. Its inertia is about a triple of that of SRM.

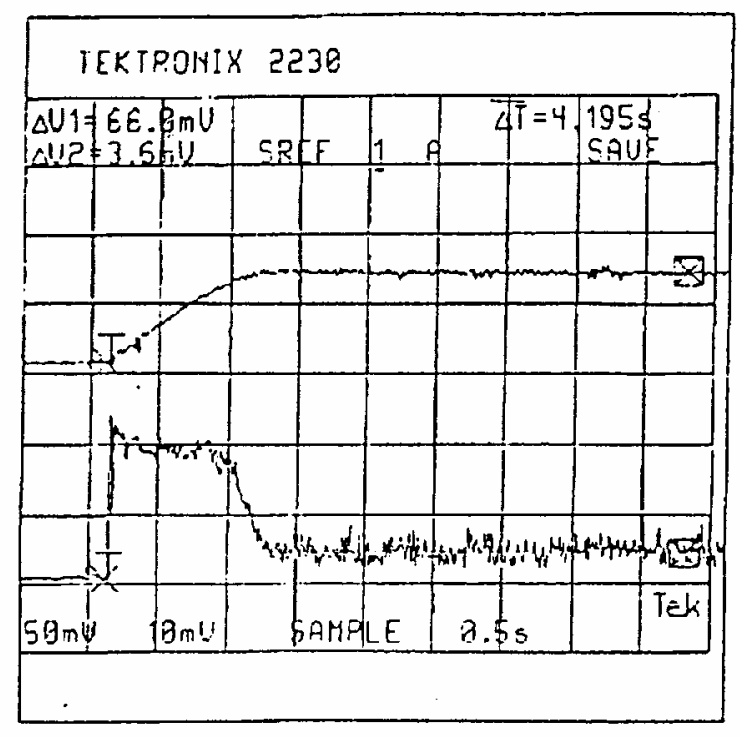

Fig. 5. Oscillograms of the speed and current, PDF-type control

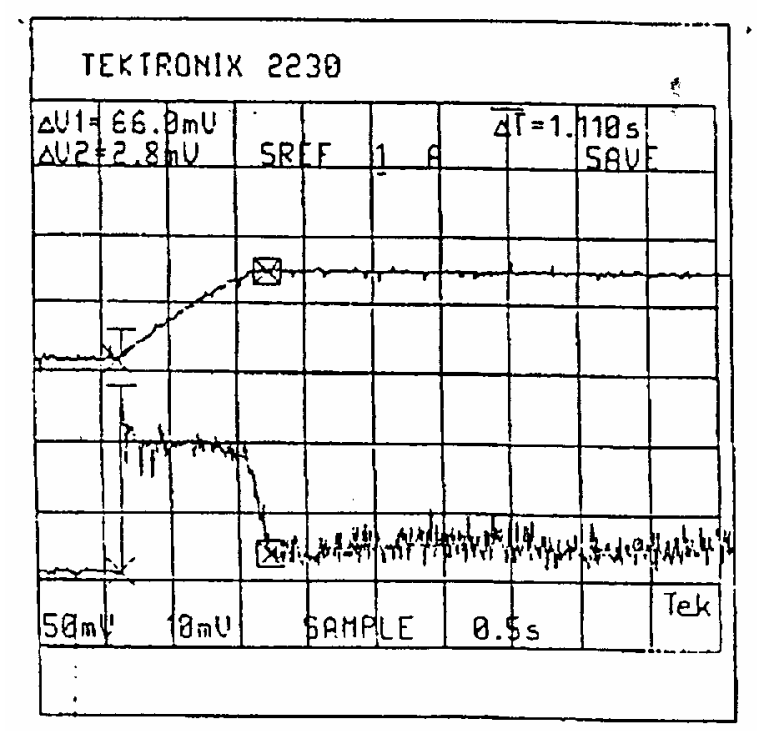

Fig. 6. Oscillograms of the speed and current, modified PDF-type control
Figs. 5 and 6 show the speed and current curves in the course of starting without current reference compensation. In all figures the upper curve is the speed $(1000 \mathrm{rpm})$, the lower one is the current flowing in the common point of stator windings $(10 \mathrm{~A} / \mathrm{div})$. Figs. 5 and 6 are related to the no-load operation mode. A PDF-type speed control [7] is applied in the case of Fig. 5, while a PDF-type speed control modified by a square-root function [8] is used in the case of Fig. 6.

\section{Conclusions}

The paper proposed a simple control method for SRM drives. The proposed ripple reduced control method changes only the turn-on and the turn-off angles depending on the speed and current reference. The modification of the current reference is suggested for a simpler four-transistor inverter. This method provides that torque ripple would not be significantly higher at a Millerkind $(n+1)$ transistor converter controlled n-phase drive in other words a reduced switching item number SRM converter feed - than at independent current control in each phases carried out with $2 n$ transistors The simulations and experimental results demonstrate that the proposed method is a promising tool to control the SRM drives.

\section{ACKNOWLEDGMENT}

This paper was supported by the Hungarian Academy of Science (Bolyai Sholarship), by the Hungarian National Scientific Funds (OTKA Ref. No.T032469) and by the Hungarian Ministry of Education (FKFP Ref. No. $0530 / 2000$ ) for which the author expresses their sincere gratitude.

\section{REFERENCES}

[1] B.K. Bose, T.J.E. Miller, "Microcomputer Control of Switched Reluctance Motor,“ IEEE/IAS Annual Meeting, 1985. pp. 542-547.

[2] T.J.E. Miller, Brushless permanent-magnet reluctance motor drives, Oxford University Press, 1989

[3] T.J.E. Miller, P.G.Bower, R.C. Becerra and M. Ehsani, "Four quadrant brushless reluctance motor drive," IEEE Conf., Power Electronics and Variable Speed Drives, July 1988, pp.273-276.

[4] T.J.E.Miller, "Switched Reluctance Motors and Their Control," Oxford, England: Oxford Press, 1993.

[5] D. Jang, I. Husain, M. Ehsani, "Modified (n+1) Switch Converter for Switched Reluctance Motor Drives," IEEE-PESC'95 Conf. Rec.,Atlanta (USA), pp.1121-1127.

[6] A. Stankovic, G. Tadmor, "On Torque Ripple Reduction in Current-Fed Switched Reluctance Motors," IEEE Transactions on Industrial Electronics, Vol. 46, No. 1, February 1999. pp. 177183.

[7] N.V. Diep, L. Szamel, "Up-to-date Control Strategy in the Regulators of Robot Drives," PEMC'90 Budapest, 1990. pp. .811815 .

[8] L. Szamel, "Ripple Reduced Control of Switched Reluctance Motor Drives," EDPE 2001, International Conference on Electrical Drives and Power Electronics, Podbanske (Slovak republic), 3-5 October 2001. pp. 48-53. 